\title{
An Emulation System for Predicting Master/Slave Program Performance ${ }^{\star}$
}

\author{
Yasuharu Mizutani, Fumihiko Ino, and Kenichi Hagihara \\ Graduate School of Information Science and Technology, Osaka University \\ 1-3 Machikaneyama, Toyonaka, Osaka 560-8531, Japan \\ \{mizutani, ino, hagihara\}@ist.osaka-u.ac.jp
}

\begin{abstract}
This paper describes the design and implementation of a performance prediction system, named Master/Slave Emulator (MSE) for message passing programs. The aim of MSE is to assist the performance study of master/slave $(\mathrm{M} / \mathrm{S})$ programs on clusters of PCs. MSE provides accurate predictions, so that enables us to analyze the performance of $\mathrm{M} / \mathrm{S}$ programs.
\end{abstract}

\section{Introduction}

Master/slave (M/S) paradigm is an adaptive programming paradigm for computing on heterogeneous systems such as clusters and grids. This paradigm provides a dynamic load-balancing mechanism, so that allows us to develop high performance programs on such heterogeneous systems. However, M/S program performance can decrease when the master is responsible for excessive slaves. Therefore, performance prediction is useful to investigate the optimal number of slaves as well as to study the performance of load-balancing strategies based on the M/S paradigm.

One approach for predicting the performance of $\mathrm{M} / \mathrm{S}$ programs is to use a theoretical analysis specific to the M/S paradigm. Although this approach is helpful in determining the number of slaves, it requires strict assumptions like that all tasks assigned by the master must be of same-size [1] and that workloads must be a representative distribution such as an exponential distribution.

Another approach is to use a realistic parallel computational model such as the LogP [2] family of models [3,45]. LogGP [3] has been validated with a wavefront application within $7 \%$ error [6]. On the other hand, to improve the accuracy of the model, LogGPS [4] and LoGPC [5] capture synchronous messages and network contention, respectively. Thus, many works have validated the accuracy of the LogP family and demonstrated accurate performance predictions with parallel programs. However, few works except CLUE [7] have validated these models with M/S programs. CLUE predicts the performance of an M/S program by executing the programs and modeling communication like LogP. Although CLUE shows accurate predictions on a 5-node SMP cluster, it leaves unclear whether its prediction is accurate enough to investigate the optimal number of slaves as well as to analyze the behavior of sophisticated M/S programs.

* This work was partly supported by the Foundation for C\&C Promotion, Network Development Laboratories, NEC, JSPS Research for the Future Program JSPS-RFTF99I00903, and JSPS Grant-in-Aid for Young Scientists (B)(15700030), for Scientific Research (C)(2)(14580374). 
The goal of this work is to develop a performance prediction system for the performance study of $\mathrm{M} / \mathrm{S}$ programs on clusters. Our target programs include not only $\mathrm{M} / \mathrm{S}$ programs where workloads can be dynamically determined but also M/S-based load-balancing strategies where the master can be dynamically generated, migrated, and structured in a hierarchical manner. To achieve this goal, we have developed an emulation system, named Master/Slave Emulator (MSE) for Message Passing Interface (MPI) programs, by incorporating following three approaches: (1) a dynamic behavior emulation by direct execution; (2) a low-overhead prediction by a realistic parallel computational model; and (3) a saturation point modeling by adapting the used model to the $\mathrm{M} / \mathrm{S}$ paradigm.

\section{Design Aspects for Predicting Master/Slave Program Performance}

\subsection{Dynamic Behavior Emulation for Performance Study}

To provide an accurate prediction for $\mathrm{M} / \mathrm{S}$-based load-balancing strategies, we have to capture the dynamic behavior of programs influenced by the strategies.

To capture this, we currently execute the target program without omitting any portion of program code. Although a few performance prediction systems omit some code by compiler analysis [8], we avoid this omission since sophisticated M/S programs contain a code for load balancing, and distinguishing the code automatically from given programs brings hard issues. For example, such programs perform task queueing as well as exchange workload information in addition to the original calculation and communication. Therefore, we have decided to use a run-time emulation approach to capture the dynamic behavior of the target programs.

\subsection{Low-Overhead Prediction for Accurate Performance Prediction}

In M/S programs, the master's performance can determine their overall performance. Therefore, to realize an accurate prediction of M/S program performance, we have to predict the master's behavior in precise. One approach for this is to simulate network in detail, however, detailed simulation requires an unpredictable large amount of computational effort, which perturbs the master's behavior. To avoid this perturbation, we have decided to use a low-overhead approach, or simulation by a realistic model such as LogGPS. LogGPS is an extension of LogGP and abstracts the communication of messages through the use of seven parameters: $L, o, g, G, P, s$, and $S$ [4].

Note here that LogGPS captures no network contention. Although other contentionbased models such as LoGPC can be used, we have selected contention-less LogGPS since it has shown to be accurate on our cluster, as presented later in Section 3. Furthermore, LogGPS has two advantages to LoGPC when network contention has little effect on the overall performance. First, LogGPS is simpler than LoGPC, so that causes less perturbation on the master. Second, the LogGPS parameters depend only on the target hardware while the LoGPC parameters depend on both the target hardware and software. For example, LoGPC requires application-specific parameters such as the message injection rate and the fraction of messages determined for every pair of processors. Deriving 
Table 1. $R T T_{P}$ : Round trip time for 1-byte message on Fast Ethernet with $P$ processors.

\begin{tabular}{|l|r|r|r|}
\hline \multirow{2}{*}{ MPI } & \multicolumn{3}{|c|}{$R T T_{P}(\mu \mathrm{s})$} \\
\cline { 2 - 4 } implementation & $P=2$ & $P=16$ & $P=64$ \\
\hline MPICH & 144.0 & 152.1 & 185.8 \\
LAM & 125.8 & 147.2 & 194.8 \\
MPICH-SCore & 95.4 & 97.6 & 99.2 \\
\hline
\end{tabular}

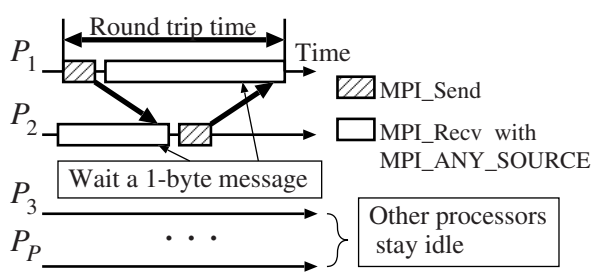

Fig. 1. Measurement of round trip time

these parameter values is complicated for some sophisticated programs [9] since such programs dynamically generate and migrate the master.

\subsection{Saturation Point Modeling for Scalability Analysis}

From our preliminary experiments, we found that the $\log \mathrm{P}$ family fail to produce the performance curve as shown later in Fig. 2. This failure is a critical problem especially when we analyze the scalability of $\mathrm{M} / \mathrm{S}$ program performance. In the following, we explain why this failure happens and how we addressed this problem.

Table 1 shows the round trip time (RTT) measured on a Fast Ethernet network by using three MPI implementations. Note here that $P-2$ of $P$ processors stay idle while the remaining two processors exchange a 1-byte message as shown in Fig. 11 In Table 1, although two common processors transmit messages for all $P$, RTT increases with $P$. This increase can strongly effect on the performance of $\mathrm{M} / \mathrm{S}$ programs since the performance can be determined by the RTT between the master and the slaves. Therefore, performance prediction for $P$ processors requires the parameter values derived from the same $P$.

However, smaller $P$ is desirable for the scalability analysis. To estimate the RTT on a large $P$ from a small $P$, we have adapted LogGPS by representing the overhead, $o=o^{\prime}+O k$, as a linear function of $P: o=o_{a}^{\prime}+o_{b}^{\prime} P+O k$, where $k$ is the message length; $o^{\prime}$ and $O$ are LogGPS constants [4]; and $o_{a}^{\prime}$ and $o_{b}^{\prime}$ are additional constants derived from two values of $o^{\prime}$ measured on a pair of a small $P$ (described in Section 3).

Our linear representation for the overhead is appropriate from the following reason. The increase of RTT has no relation to network contention since the message length and the communication pattern are fixed for all $P$ during the RTT measurement. It is due to the increase of the overhead required for retrieving arrival messages. For example, MPICH's MPI_Recv calls a select system call and Linux/FreeBSD's select retrieves the arrival-state sockets by the linear search. Since the number of sockets is equal to $P-1, o$ increases with $P$, and thereby RTT increases with $P$.

\section{Experimentation}

To validate our MSE on its prediction accuracy, we applied it to an M/S program: a parallel mandelbrot set explorer (MASE) for fractal visualization which solves a set of independent tasks whose workloads are dynamically determined. A task of MASE corresponds to test whether a point on the complex plane is included in the mandelbrot 
Table 2. LogGPS parameter values for MPICH on Fast Ethernet, derived from 8 processors. $P$ and $k$ represent the number of processors and the length for messages, respectively.

\begin{tabular}{|c|c|c|c|c|}
\hline & $L(\mu \mathrm{s})$ & $G(\mu \mathrm{s})$ & $o$ for MPI_Send $(\mu \mathrm{s})$ & $o$ for MPI_Recv $(\mu \mathrm{s})$ \\
\hline MSE & 500 & 00268 & $12.1+0.182 P+0.0708 k$ & $12.1+0.182 P+0.0722 k$ \\
\hline LogGPS & 50.0 & 0.0268 & $+0.0706 k$ & $+0.0723 k$ \\
\hline
\end{tabular}

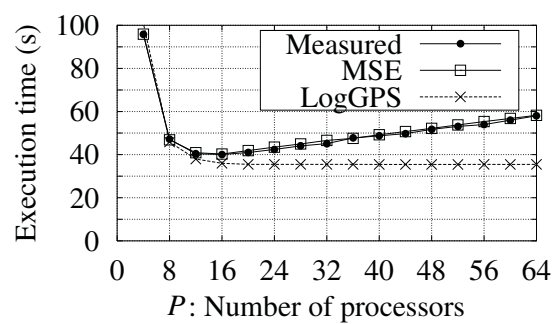

Fig. 2. Measured and predicted execution time for single master implementation (SI).

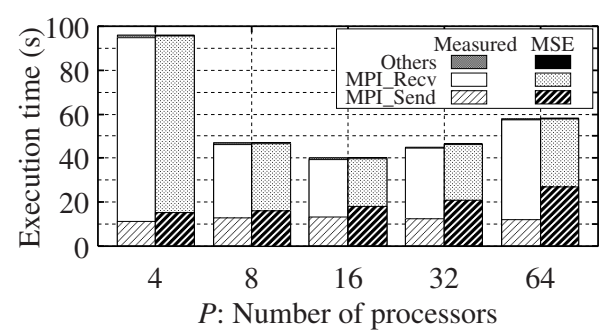

Fig. 3. Breakdown analysis of master's execution time (SI).

set. We implemented them in three variations: single (SI), multiple (ML), and dynamic (DY) master implementations (described later in Section 3.2).

We used a 64-node cluster with Pentium III $1 \mathrm{GHz}$ processors for experiments. Each node in the cluster connects to Myrinet and Fast Ethernet switches, yielding full-duplex bandwidth of $2 \mathrm{~Gb} / \mathrm{s}$ and $100 \mathrm{Mb} / \mathrm{s}$, respectively.

In the experiments, we emulated MPICH programs on Fast Ethernet by executing MPICH-SCore [10] programs on Myrinet with the same $P$ processors. Note here that MSE currently requires a high-speed network for emulation and this network must be faster than the target network. This is due to the lack of a virtual time mechanism [7], however, this is no critical problem to validate the prediction accuracy of MSE.

Table 2 shows the LogGPS parameter values for MPICH on Ethernet, derived from 8 processors. Here, we only show the values for asynchronous messages since MASE transmitted no synchronous message. Besides, we disregarded $g$ since $g$ is encapsulated in $o$ on current machines [4,5]. To derive $o_{a}^{\prime}$ and $o_{b}^{\prime}$, we first derived two LogGPS constants, $o^{\prime}$ on $P=2$ and $P=8$, in accordance with [4], then solved a pair of equations in $o_{a}^{\prime}$ and $o_{b}^{\prime}$ variables. As shown in Table 2 the coefficient of $P$ has significant influence on $o$. For example, for $k=1$, $o$ doubles when $P$ increases from 2 to 66 processors.

\subsection{Validating Prediction Accuracy}

To validate the saturation point modeling of MSE, we predicted the performance with the minimum task granularity, where the master became a bottleneck. Fig. 2] shows the measured and the predicted execution time for the SI implementation. Our MSE shows an accurate prediction within $3 \%$ error. It also shows the performance saturation point in precise, so that the optimal $P$ obtained by MSE agrees with the measured results, $P=16$. However, LogGPS fails to show the performance curve, so that drops its accuracy as $P$ increases. This failure can be explained as follows. Since MASE performs 1,048,576 round trip communications between the master and the slaves, the master takes at least 


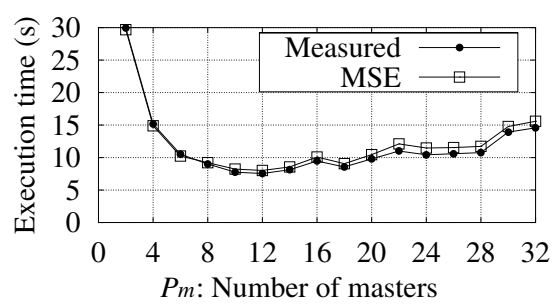

(a) ML: Multiple master implementation

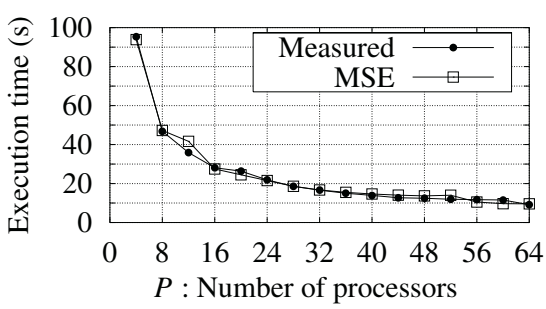

(b) DY: Dynamic master implementation

Fig. 4. Measured and predicted execution time for MASE.

$1,048,576 \cdot 2 o \mu \mathrm{s}$. Substituting $P=8$ and $P=64$ to $o$ (see Table 2) and subtracting them gives $1,048,576 \cdot 2 \cdot 0.182 \cdot(64-8) / 10^{6}=21.4 \mathrm{~s}$, or the contribution of our linear overhead when $P=64$. This value is close to $22.5 \mathrm{~s}$, or the difference between the measured and the LogGPS time when $P=64$. Thus, our linear overhead representation is necessary for the scalability analysis for $\mathrm{M} / \mathrm{S}$ programs since it can significantly effect on the performance on large $P$.

Fig. 3 shows the breakdown of the master's execution time. We can see that MSE estimates the total time in precise for all $P$, however, with the increase of $P$, it estimates longer time for MPI_Send and shorter time for MPI_Recv. This discrepancy is due to our simplified model, which assumes that both the send and the receipt overheads require the same $o^{\prime}$. That is, to simplify our model, we have made the assumption, but the actual send overhead contains no cost proportional to $P$. Our assumption works fine for $\mathrm{M} / \mathrm{S}$ programs where assigned tasks and their results are unredirected. However, for $\mathrm{M} / \mathrm{S}$ programs where the master migrates before receiving the results of assigned tasks, the total number of the master's MPI_Send differs to that of its MPI_Recv, so that we have to avoid this simplification. One solution for this is to distinguish $o_{a}^{\prime}$ and $o_{b}^{\prime}$ between the send and the receipt overheads.

\subsection{Validating Dynamic Behavior Emulation}

We applied our MSE to two sophisticated variations of MASE. One is the ML implementation, in which each of $P_{m}$ masters has $P / P_{m}$ responsible slaves, where $2 \leq P_{m} \leq P / 2$. The other is the DY implementation, in which the number of masters can change during program execution. In DY, every master can split its responsible tasks and slaves in two groups and assign one group to a responsible slave. The assigned slave then becomes the master of the half slave and works as a secondary master until it completes the assigned tasks. The splitting and merging of the master are triggered by the statistics of the slaves' wait time from the request of a task until its assignment.

Fig. 4(a) shows the results for ML on $P=64$. In Fig. (4)a), although the measured time appears as an irregular graph, our MSE presents an accurate prediction within 10\% error. This good accuracy comes from our emulation approach since it realizes almost the same workload distributions as the original. Actually, the measured time increases when $P=16$ due to the splitting of tasks with imbalanced workloads.

Fig.4(b) shows the results for DY. Although DY behaves in a more complex manner, MSE shows a similar performance to the measured results. From the above study, we 
can conclude that ML gives the best performance among the three implementations if we can detect the optimal number of $P_{m}$. Thus, MSE successfully shows the irregular performance of M/S programs, which is not easy for static and post-mortem approaches.

\section{Conclusion}

We have presented the design and implementation of a performance prediction system, named MSE, which aims at assisting the performance study of MPI programs based on the M/S paradigm. Our MSE employs a best effort emulation method and a realistic parallel computational model, the LogGPS model. It also adapts LogGPS to the M/S paradigm by capturing the overhead required for retrieving messages arrived from slaves. MSE has demonstrated an accurate prediction within 10\% error for a 64-node cluster. It also has shown that our linear overhead representation is necessary for M/S programs, especially on a large number of processors.

\section{References}

1. Beaumont, O., Legrand, A., Robert, Y.: The master-slave paradigm with heterogeneous processors. In: Proc. 3rd IEEE Int'l Conf. on Cluster Computing. (2001) 419-426

2. Culler, D.E., Karp, R.M., Patterson, D.A., Sahay, A., Schauser, K.E., Santos, E., Subramonian, R., von Eicken, T.: LogP: Towards a realistic model of parallel computation. In: Proc. 4th ACM SIGPLAN Symp. on Principles Practice of Parallel Programming. (1993) 1-12

3. Alexandrov, A., Ionescu, M.F., Schauser, K.E., Scheiman, C.: LogGP: Incorporating long messages into the LogP model for parallel computation. J. of Parallel and Distributed Computing 44 (1997) 71-79

4. Ino, F., Fujimoto, N., Hagihara, K.: LogGPS: A parallel computational model for synchronization analysis. In: Proc. 8th ACM SIGPLAN Symp. on Principles and Practice of Parallel Programming. (2001) 133-142

5. Moritz, C.A., Frank, M.I.: LoGPC: Modeling network contention in message-passing programs. IEEE Trans. on Parallel and Distributed Systems 12 (2001) 404-415

6. Adve, V.S., Bagrodia, R., Browne, J.C., Deelman, E., Dube, A., Houstis, E.N., Rice, J.R., Sakellariou, R., Sundaram-Stukel, D.J., Teller, P.J., Vernon, M.K.: POEMS: End-to-end performance design of large parallel adaptive computational systems. IEEE Trans. on Software Engineering 26 (2000) 1027-1048

7. Kvasnicka, D.F., Hlavacs, H., Ueberhuber, C.W.: Simulating parallel program performance with CLUE. In: Proc. 2001 Int'1 Symp. on Performance Evaluation of Computer and Telecommuication Systems (SPECTS'01). (2001) 140-149

8. Adve, V.S., Bagrodia, R., Deelman, E., Sakellariou, R.: Compiler-optimized simulation of large-scale applications on high performance architectures. J. of Parallel and Distributed Computing 62 (2002) 393-426

9. Czarnul, P., Tomko, K., Krawczyk, H.: Dynamic partitioning of the divide-and-conquer scheme with migration in PVM environment. In: Proc. 8th European PVM/MPI Users' Group Meeting (EuroPVM/MPI'01). (2001) 174-182

10. O'Carroll, F., Tezuka, H., Hori, A., Ishikawa, Y.: The design and implementation of zero copy MPI using commodity hardware with a high performance network. In: Proc. 12th ACM Int'1 Conf. on Supercomputing, http://www.pccluster.org/(1998) 243-250 